\title{
DROIT ET LIBERTÉ DANS LES SOCIÉTÉS SANS ÉTAT : UNE RELECTURE DE PIERRE CLASTRES À LA LUMIÈRE D’UNE APPROCHE MICROSCOPIQUE DU DROIT
}

\section{Maxime de Brogniez}

Université Saint-Louis - Bruxelles | Revue interdisciplinaire d'études juridiques » 2021/1 Volume 86 | pages 5 à 36

ISSN 0770-2310

Article disponible en ligne à l'adresse :

https://www.cairn.info/revue-interdisciplinaire-d-etudesjuridiques-2021-1-page-5.htm

Distribution électronique Cairn.info pour Université Saint-Louis - Bruxelles.

(C) Université Saint-Louis - Bruxelles. Tous droits réservés pour tous pays.

La reproduction ou représentation de cet article, notamment par photocopie, n'est autorisée que dans les limites des conditions générales d'utilisation du site ou, le cas échéant, des conditions générales de la licence souscrite par votre établissement. Toute autre reproduction ou représentation, en tout ou partie, sous quelque forme et de quelque manière que ce soit, est interdite sauf accord préalable et écrit de l'éditeur, en dehors des cas prévus par la législation en vigueur en France. Il est précisé que son stockage dans une base de données est également interdit. 
R.I.E.J., 2021.86

\title{
ÉTUDES
}

\section{Droit et liberté dans les sociétés sans État: une relecture de Pierre Clastres à la lumière d'une approche microscopique du droit}

\author{
Maxime DE BROGNIEZ \\ Assistant doctorant à l'Université de Liège
}

\begin{abstract}
Résumé
La théorie microscopique du droit élaborée par Lucien François lève l'incurable ambivalence sémantique du mot "droit» en repensant le phénomène juridique à partir de sa manifestation minimale, le jurème. Les outils fournis par Lucien François permettent de porter un regard neuf sur les sociétés sans État, sociétés que Pierre Clastres qualifie de sociétés contre l'État. À partir des travaux de Pierre Clastres, nous montrerons que ces sociétés, bien que sans État, ne sont pas a-juridiques pour autant. II existe des rapports juridiques qui prennent une forme très particulière, celle du cercle d'exigence mutuelle. Cette figure, ainsi que celle du nimbe - également thématisée par Lucien François -, permettront de questionner la notion de liberté dans ces sociétés. Si celle-ci peut au premier abord paraître radicalement opposée à nos conceptions, la réalité est sans doute moins binaire.
\end{abstract}

\section{Abstract : Law and Liberty in Stateless Societies: a Reading of Pierre Clastres's Work through a Microscopic Legal Theory}

Lucien François's microscopic legal theory avoids a persistent semantical ambiguity by rethinking the concept of law through its smallest unit, the jurème. The tools provided by Lucien François enable us to adopt a new perspective on stateless societies, societies described by Pierre Clastres as societies against the State. Based on Pierre Clastres's work, we show that these societies, even though they are stateless, are not a-juridical. Some legal relationships take a very peculiar form, that of the cercle d'exigence mutuelle. That concept, and that of the nimbe - also coined by Lucien François enables us to reconsider the notion of liberty in these societies. If it may at first sight seem radically opposed to our ideas, the reality is perhaps less binary. 


\section{Introduction}

Traditionnellement, le droit est présenté comme une émanation de l'État. Ainsi, les sociétés sans État sont généralement qualifiées de sociétés a-juridiques. À travers les travaux de l'anthropologue et ethnologue français Pierre Clastres et ceux du théoricien belge du droit Lucien François, nous montrerons que ces sociétés sans État ne sont pas, pour autant, a-juridiques. Les outils fournis par Lucien François nous permettront d'analyser, dans une première partie, la forme très particulière que prennent les rapports juridiques dans les sociétés sans État. En assurant une égalité stricte entre les membres de la « tribu », cette configuration permet, selon Pierre Clastres, d'empêcher l'émergence de l'État. Si ces sociétés exigent une égalité parfaite entre leurs membres c'est, écrit Pierre Clastres, pour préserver leur liberté ${ }^{1}$. Cette affirmation nous conduira, dans une seconde partie, à examiner la notion de liberté dans les sociétés dites primitives. Avant de conclure, un détour par les travaux de Marshall Sahlins, Raymond Firth et Lucien François permettra de nuancer l'approche de Pierre Clastres et de poser la question du caractère conjoncturel, voire culturel, de la liberté.

La confrontation de deux disciplines, l'anthropologie et la théorie $d u$ droit (en ce que celle-ci s'intéresse à ce qu'est le droit ${ }^{2}$ ), nous permettra de jeter un éclairage neuf sur des concepts fondamentaux pour la philosophie $d u$ droit. Ainsi, les rapports entre droit et contrainte d'une part et, d'autre part, entre droit, liberté et État se verront questionnés. Le choix des auteurs étudiés est, du reste, guidé par l'ambition de montrer qu'une analyse positiviste d'un phénomène normatif qui, par son ancrage résolument mythique et religieux, pourrait trop rapidement être rangé du côté du droit naturel est possible, et même souhaitable, en ce qu'elle remet en perspective les fondements des ordres juridiques étatiques proprement dits. S'il est vrai, comme l'écrit Émile Durkheim, qu'« on n'explique qu'en comparant $»^{3}$, l'étude qui va suivre se veut avant tout mise en rapport d'objets a priori hétérogènes mais qui, pensés ensemble, permettent une meilleure compréhension de leurs enjeux respectifs. Ainsi, notre propos n'est pas de questionner la validité ou la

\footnotetext{
${ }^{1}$ Voy. infra ; P. CLASTRES, "Entretien avec L'Anti-Mythes ", in Pierre Clastres, M. Abensour et A. Kupiec (dir.), Paris, Sens \& Tonka, 2011, p. 37.

2 "Théorie, elle se propose uniquement et exclusivement de connaître son objet, c'est-à-dire d'établir ce qu'est le droit et comment il est »(H. KELSEN, Théorie pure du droit, trad. fr. Ch. Eisenmann, Paris, Dalloz, $2^{\mathrm{e}}$ éd., 1962, p. 1).

${ }^{3}$ É. DuRKHEIM, Le Suicide, Paris, Librairie Félix Alcan, 1930, p. 1.
} 
pertinence des analyses de Clastres ${ }^{4}$ ou de François pour elles-mêmes, mais bien d'opérer des associations fécondes. Dans un tout autre domaine, Georges Didi-Huberman écrit: "L'imagination accepte le multiple et le reconduit sans cesse pour y déceler de nouveaux "rapports intimes et secrets", de nouvelles "correspondances et analogies" qui seront ellesmêmes inépuisables comme est inépuisable toute pensée des relations qu'un montage inédit, à chaque fois, sera susceptible de manifester $»^{5}$. Dans le domaine de la philosophie du droit, l'imagination doit, certes, rapidement céder la place à la rigueur et, ainsi, permettre de mieux voir «ce que ces rapprochements ont d'instructif $»^{6}$.

\section{Des sociétés parfaitement égalitaires ?}

En 1974, Pierre Clastres publie un livre qui, du point de vue de la théorie politique, fera date : La Société contre l'État. «Sauvages d'avant la civilisation, peuples d'avant l'écriture, sociétés d'avant l'Histoire : elles sont certes les bien nommées, ces sociétés primitives, sociétés premières de se déployer dans l'ignorance de la division, premières d'exister avant le fatal malencontre ${ }^{[7]}$. Objet privilégié, sinon exclusif de l'ethnologie : les sociétés sans État $\|^{8}$. Dépassant le simple constat de l'absence du phénomène étatique dans ces sociétés, Clastres, fort de ses diverses expériences de terrain - principalement chez les Indiens guyaki du Paraguay, mais aussi chez les Guarani Chiripa ou encore les Javae -, juge « que la société primitive [s'est] édifiée comme société contre l'État, qu'elle [a] monté un système de défense pour rendre impossible la formation d'un pouvoir qui, détaché de la communauté, aurait la liberté de se retourner contre elle, d'asservir ses membres à un chef $\|^{9}$. II s'oppose au discours dominant selon lequel « les sociétés primitives sont privées de quelque chose - l'État - qui leur est, comme à toute autre société - la nôtre par exemple - nécessaire ${ }^{10}$; selon lequel ces sociétés seraient incomplètes, « [subsisteraient] dans l'expérience

\footnotetext{
${ }^{4}$ Pour une critique des analyses de CLASTRES voy. J.-W. LAPIERRE, Vivre sans État ? Essai sur le pouvoir politique et l'innovation sociale, Paris, Seuil, 1977, p. 323 et s.

${ }^{5} \mathrm{G}$. DIDI-Huberman, Atlas ou le gai savoir inquiet, Paris, Les éditions de Minuit, 2011, p. 14.

${ }^{6}$ É. DURKHEIM, op. cit., p. 7.

${ }^{7}$ « Malencontre : accident tragique, malchance inaugurale dont les effets ne cessent de l'amplifier au point que s'abolit la mémoire de l'avant, au point que l'amour de la servitude s'est substitué au désir de liberté » (P. CLASTRES, "Liberté, malencontre, innommable », postface à É. DE LA BoÉTIE, Discours de la servitude volontaire, Paris, Payot, 1978, p. 231).

${ }^{8}$ Ibidem, p. 233.

${ }^{9}$ Cl. LEFORT, «L'œuvre de Clastres », in L'esprit des lois sauvages, M. Abensour (dir.), Paris, Seuil, 1987, p. 191.

${ }^{10}$ P. ClAStRES, La Société contre l'État, Paris, Les éditions de Minuit, 1974, p. 161.
} 
peut-être douloureuse d'un manque - manque d'État - qu'elles tenteraient, toujours en vain, de combler ${ }^{11}$; selon lequel " on ne peut pas penser la société sans l'État, l'État est le destin de toute société $~^{12}$. Clastres s'attache à démontrer l'ethnocentrisme de cette conception à sens unique de l'Histoire ${ }^{13}$.

\section{A. Des sociétés sans État et donc sans droit ?}

\section{Des sociétés sans État...}

D'emblée, à contre-courant de l'analyse marxiste, Clastres pose la prééminence du politique sur l'économique. La division première n'est pas celle qui oppose les exploiteurs aux exploités mais celle qui met aux prises « ceux qui commandent et ceux qui obéissent, (...) ceux qui ont le pouvoir et ceux qui subissent le pouvoir ${ }^{14}$. II est impossible, selon Clastres, de voir émerger une division entre riches et pauvres dans une société où tout fonctionnerait pour empêcher la division en général : "C'est l'État qui engendre les classes ${ }^{15}$.

D'où viendrait alors l'État? Quelles sont les conditions de sa genèse ? On ne trouve pas de théorie de la naissance de l'État dans l'œuvre de Clastres. "S'il paraît encore impossible de déterminer les conditions d'apparition de l'État, on peut en revanche préciser les conditions de sa nonapparition ${ }^{16}$. Clastres en identifie deux, qui seraient au service d'un impératif plus général d'égalité, garantie de la non-émergence de l'État.

La première condition est l'absence de Roi, l'absence de chef ou, plus exactement, l'absence de chef disposant d'un pouvoir coercitif. A contrario, la présence d'un chef sans pouvoir est requise. Les sociétés contre l'État disposent bien d'un chef, mais celui-ci est privé de tout pouvoir effectif ${ }^{17}$. L'existence de la chefferie, d'un lieu du pouvoir, est en quelque sorte nécessaire pour empêcher l'émergence effective de ce dernier: "pour empêcher que ce pouvoir devienne réel, il faut piéger ce lieu, il faut y mettre quelqu'un, et ce quelqu'un c'est le chef ${ }^{18}$. Vis-à-vis de la communauté, le

\footnotetext{
${ }^{11}$ Ibidem

12 Ibidem

${ }^{13}$ Sur l'évolutionnisme en anthropologie, voy. N. RoULAND, L'Anthropologie juridique, Paris, PUF, 1990 , p. 14 et s.

${ }^{14}$ P. ClASTRES, La Société contre l'État, op. cit., p. 161.

15 Ibidem, p. 15.

${ }^{16}$ Ibidem, p. 175.

17 Voy. en particulier le second chapitre de La Société contre l'État : "Échange et pouvoir : philosophie de la chefferie indienne " (p. 25-42).

${ }^{18}$ P. ClAstres, « Entretien avec L'Anti-Mythes », op. cit., p. 29.
} 
chef n'a aucun pouvoir contraignant. Vis-à-vis de prétentions extérieures, la chefferie permet d'opposer un lieu du pouvoir ineffectif, certes, mais occupé.

La guerre ou, plus précisément, la guerre comme outil de limitation démographique, constitue la deuxième condition de non-apparition de l'État. Si l'on écrit souvent que "l'extrême morcellement sous lequel se présente partout la société primitive serait la cause (...) de la fréquence de la guerre dans ce type de sociétés ${ }^{19}$, " ce n'est pas la guerre qui est l'effet du morcellement, c'est le morcellement qui est l'effet de la guerre. II n'en est pas seulement l'effet mais le but : la guerre est à la fois la cause et le moyen d'un effet et d'une fin recherchés, le morcellement de la société primitive $»^{20}$. Contrairement à la logique étatique, toujours nataliste et expansionniste ${ }^{21}$, la société "primitive » cherche à limiter sa croissance démographique. Les sociétés "premières", en effet, "connaissent et mettent en œuvre de multiples moyens de contrôler ou d'empêcher la croissance de leur population : avortement, infanticide, tabous sexuels, sevrage tardif, etc. ${ }^{22}$. II serait faux, d'après Clastres, de justifier cette volonté de limitation démographique par une économie de subsistance, inapte à subvenir aux besoins d'une population trop nombreuse. Les sociétés dites primitives " démontrent une capacité de satisfaire leurs besoins au moins égale à celle dont s'enorgueillit la société industrielle et technicienne $»^{23}$. Ces sociétés se contentent de produire ce dont elles ont besoin en un temps aussi réduit que possible. On peut parler de sociétés de loisir, de sociétés de refus du travail ${ }^{24}$. Cette logique, étrange aux yeux de l'observateur " civilisé ", est - encore un positionnement de lutte contre l'émergence de l'État : " lorsque disparaît le refus du travail, lorsqu'au sens du loisir se substitue le goût de l'accumulation, lorsqu'en un mot se fait jour dans le corps social (...) cette force sans laquelle les Sauvages ne renonceraient pas au loisir et qui détruit la société en tant que société primitive : cette force, c'est la puissance de contraindre, c'est la capacité de coercition, c'est le pouvoir politique $»^{25}$. Ce qui, en réalité, explique la volonté de maintenir la démographie de la société " primitive " dans des limites resserrées est que "les choses ne peuvent fonctionner selon le modèle primitif que si les gens sont peu nombreux. Ou,

\footnotetext{
${ }^{19}$ P. CLASTRES, Archéologie de la violence. La guerre dans les sociétés primitives, La Tour d'Aigues, Éditions de l'Aube, 2013, p. 35.

${ }^{20}$ Ibidem.

${ }^{21}$ P. CLASTRES, « Entretien avec L'Anti-Mythes », op. cit., p. 38.

22 P. ClASTRES, La Société contre l'État, op. cit., p. 69.

${ }^{23}$ Ibidem, p. 163.

${ }^{24}$ À ce sujet, voy. aussi M. SAHLINS, Âge de pierre, âge d'abondance. L'économie des sociétés primitives, Paris, Gallimard, 1976.

${ }_{25}$ P. CLASTRES, La Société contre l'État, op. cit., p. 167-168.
} 
en d'autres termes, pour qu'une société soit primitive, il faut qu'elle soit petite par le nombre $»^{26}$. C'est cela qui justifie le morcellement comme but et non comme cause de la guerre.

Ces deux conditions de non-émergence de l'État - maîtrise structurale du pouvoir et limitation démographique - sont au service d'un axiome plus fondamental : l'égalité comme impératif absolu, " l'interdiction, non formulée mais dite cependant, de l'inégalité $»^{27}$. Tous les processus décrits comme visant à lutter contre l'apparition de l'État ont en dernière instance pour objectif d'empêcher l'émergence de l'inégalité. Ainsi, écrit Clastres, " sera déterminée comme société primitive toute machine sociale qui fonctionne selon l'absence de la relation de pouvoir. Sera par conséquent dite à État, toute société dont le fonctionnement implique, si minime puisse-t-il nous paraître, l'exercice du pouvoir ${ }^{28}$. Cette stricte correspondance entre inégalités et société à État d'une part et, d'autre part, entre égalité et société sans État mérite d'être nuancée. L'impératif d'égalité est sans doute avant tout un idéal - c'est-à-dire un horizon guidant la vie et les décisions des Indiens indépendamment de sa pleine effectivité, une norme connaissant des écarts. Bien que l'archéologie et l'anthropologie enseignent que l'émergence des inégalités dans une société est bien chronologiquement et logiquement antérieure à sa structuration comme État ${ }^{29}$, il n'empêche que cette « règle » a connu des exceptions et que " parfois, un autre monde a été possible ${ }^{30}$.

\footnotetext{
${ }^{26}$ Ibidem, p. 181.

${ }^{27}$ Ibidem, p. 169.

${ }^{28}$ P. CLASTRES, « Liberté, malencontre, innommable », op. cit., p. 234.

29 C'est au Néolithique, vers 4500 avant notre ère, qu'émergent les premières sociétés inégalitaires. "Tout comme le Néolithique lui-même, le développement des inégalités a résulté d'une pluralité de facteurs, avec des conditions de possibilité d'ordre matériel - économie de production, poussée démographique, pièges territoriaux - et des conditions d'ordre idéel culturelles, idéologiques, voire psychosociales. De fait, toutes les sociétés néolithiques ont débouché sur des sociétés inégalitaires puis, à plus ou moins brève échéance, sur des sociétés étatiques et urbaines » (J.-P. DEMOULE, « Naissance des inégalités et prémisses de l'État », in La Révolution néolithique dans le monde, J.-P. Demoule (dir.), Paris, CNRS éditions, 2009, p. 424).

${ }_{30} \mathrm{Ibidem}$. Maurice GODELIER prend l'exemple des Baruya parmi lesquels il a vécu en PapouasieNouvelle-Guinée à partir de 1967. Les Baruya n'avaient pas de chef. Pourtant, « il existait une double hiérarchie. La première entre les hommes et les femmes et entre tous les individus selon leur âge » (M. GODELIER, "Tribus et États. Quelques hypothèses », in La Révolution néolithique dans le monde, op. cit., p. 428). Cette hiérarchie, qui du reste existe également dans les sociétés étudiées par CLASTRES, découlait de rituels d'initiation. "C'est alors qu'une seconde hiérarchie intervenait, celle entre les clans qui possédaient les objets rituels et les formules secrètes permettant d'initier les hommes et d'en faire des guerriers ou des chamans et les autres clans » (ibidem). Il y avait donc des individus dont la parole comptait plus que celle des autres et auxquels le reste du groupe était redevable de dons de biens et de services. Ces individus étaient toutefois toujours tenus de produire leurs propres moyens de subsistance. L'État n'a pu apparaître que lorsque certains groupes « ont commencé à consacrer pleinement leur existence et leur temps à
} 
Les règles qui gouvernent les Indiens et qui expliquent l'impératif d'égalité trouvent leur fondement dans des mythes très anciens - des missionnaires les ont transcrits dès le $\mathrm{XV}^{\mathrm{e}}$ siècle - que les karai (prophètes) rappellent régulièrement. "La subsistance de la société guarani, c'est son monde religieux écrit Clastres. Que se perde pour eux l'ancrage en ce monde : alors la société s'écroulera. Le rapport des Guarani à leurs dieux est ce qui les maintient comme Soi collectif, ce qui les rassemble dans une communauté de croyants. Cette communauté ne survivrait pas un instant à la perte de la croyance. Les Indiens le savent bien ${ }^{31}$. Sans prétendre dresser un panorama exhaustif de la mythologie guarani ${ }^{32}$, il est indispensable de s'attarder brièvement sur la dialectique de l'Un et du multiple qu'elle met en œuvre pour mieux comprendre le fondement de l'exigence d'égalité : «Les choses en leur totalité sont une ; et pour nous qui n'avons pas désiré cela, elles sont mauvaises » disent les Indiens ${ }^{33}$. Les hommes habitent une terre mauvaise et cette imperfection vient de ce que "les choses en leur totalité sont une $»^{34}$. L'Un, explique Clastres, c'est l'Imparfait, c'est le Mal. Dès lors, " quel est-il ce non-Un si obstinément désiré des Guarani ? » ${ }^{35}$. II faut se garder d'assimiler l'Un au Tout. L'Un existe sur le mode du transitoire, du passager, de l'éphémère. II qualifie l'incomplet. Dans la Terre sans Mal à laquelle aspirent les Indiens, " rien de ce qui existe ne peut être dit Un $»^{36}$. Ainsi, ses habitants ne sont ni seulement des hommes, ni seulement des dieux, mais bien les deux à la fois. "Le Bien, ce n'est pas le multiple, c'est le deux, à la fois l'un et son autre, le deux qui désigne véridiquement les êtres complets. Ywy mara eÿ, destination des Derniers Hommes, n'abrite plus d'hommes, n'abrite plus de dieux: seulement des égaux, dieux-hommes, hommes-dieux, tel que nul d'entre eux ne se dit selon l'Un $»^{37}$. Ce rapport intime des Indiens avec leur part divine se vit collectivement. "Entre le Moi de l'individu et le Soi des dieux, il y a la communauté des hommes, il y a la

\footnotetext{
l'accomplissement de fonctions [politiques et religieuses] qui légitimaient, aux yeux des autres groupes composant avec eux une société, d'une part leur droit de ne plus produire eux-mêmes leurs conditions concrètes d'existence et d'autre part celui de contrôler l'accès des autres membres de la société aux conditions mêmes de la production des moyens matériels de leur existence sociale, et enfin leur droit de s'approprier l'usage de leur force de travail ainsi qu'une partie des biens et des services produits par leur travail » (ibidem, p. 435).

${ }^{31}$ P. ClASTRES, Le Grand parler. Mythes et chants sacrés des Indiens Guarani, Paris, Seuil, 1974, p. 8.

${ }^{32}$ On se rapportera pour cela à P. CLASTRES, Le Grand parler..., op. cit.

${ }^{33}$ P. ClASTRES, La Société contre l'État, op. cit., p. 146.

${ }^{34}$ Ibidem, p. 147.

${ }^{35}$ Ibidem.

${ }^{36}$ Ibidem, p. 149.

${ }^{37}$ Ibidem.
} 
tribu $»^{38}$. Les Guarani sont élus des dieux « en tant qu'ils appartiennent à la tribu, laquelle découvre le fondement de sa socialité dans la conscience qu'elle a d'elle-même comme lieu de la Parole issue des dieux. À l'origine du tribalisme guarani se trouve la divinité de l'ayvu: l'être social de la tribu s'enracine dans le divin ${ }^{39}$. Sous l'équation métaphysique qui égale le Mal à l'Un, Clastres en décèle une autre, « plus secrète, et d'ordre politique, qui dit que l'Un, c'est l'État ${ }^{40}$. À une relation où trois termes - « indiens - dieux tribu »-s'impliquent mutuellement sur le mode du multiple, le triomphe de l'Un substitue un pouvoir centralisé et séparé. L'Un, c'est l'« essence universelle de l'État $»^{41}$.

L'inégalité, la relation de domination, le pouvoir politique, contiennent en germe la structure étatique. Leur émergence impliquerait ipso facto un ébranlement de la sociabilité des Indiens et du fondement mythique de leur pensée : " si "ça marchait" [si le chef se servait de la société pour réaliser des fins privées] alors on aurait là le lieu natal du pouvoir politique comme contrainte et violence, on aurait la première incarnation, la figure minimale de l'État $\nu^{42}$. Entre le chef qui exige de ses sujets qu'ils lui versent une partie de leurs récoltes et l'administration fiscale, il y aurait, si l'on suit Clastres, une différence de degré mais non de nature.

\section{2. ... donc sans droit ?}

Traditionnellement, ces sociétés sans État, sans organisation politique centralisée, sont présentées comme a-juridiques. Elles n'ont, dit-on, pas de droit $^{43}$. Si d'aucuns ont au contraire entrepris de soutenir que l'absence de

\footnotetext{
${ }^{38}$ P. ClAStRES, Le Grand parler..., op. cit., p. 27.

39 Ibidem.

Clastres commente le fragment suivant:

"Connu le fondement de la Parole future,

en son divin savoir des choses,

savoir qui déploie les choses,

il sait alors pour lui-même

la source de ce qui est destiné à rassembler.

La terre n'existe pas encore,

règne la nuit originaire,

il n'y a pas de savoir des choses :

du savoir qui déploie les choses,

il sait alors pour lui-même

la source de ce qui est destiné à rassembler » (ibidem, p. 26).

${ }^{40}$ P. ClASTRES, La Société contre l'État, op. cit., p. 184.

41 Ibidem.

42 Ibidem, p. 178.

${ }^{43}$ R. JACOB, "Le droit, l'anthropologue et le microscope ", in Le Droit sans la justice. Actes de la rencontre du 8 novembre 2002 autour du Cap des Tempêtes de Lucien François, E. Delruelle et
} 
pouvoir centralisé n'impliquait pas l'absence de tout phénomène juridique, celui-ci n'en est pas moins le plus souvent appréhendé, alors, via des concepts alternatifs tels que modèle, structure, règle, coutume ou encore norme $e^{44}$. Néanmoins, ces phénomènes, faute d'un législateur étatique ${ }^{45}$, faute de lois écrites ${ }^{46}$, faute d'organisation judiciaire ${ }^{47}$, sont, dans une vision largement partagée, réduits à n'être que des pré-droits, des quasi-droits ${ }^{48}$ ou encore d'autres systèmes de droits ${ }^{49}$. La diversité des critères avancés pour dégager un seuil de juridicité reflète, il est vrai, la fantaisie qui précède à leur énonciation. Lorsqu'un lien est tout de même établi entre norme et droit, il reste malaisé de définir le critère de rattachement de la première au second. Ainsi, pour l'Américain Karl Nickerson Llewellyn, la « "saveur en plus" du droit est ce qui le distingue des autres facteurs de régulation sociale $»^{50}$. Outre la difficulté de dire si une norme ou une coutume est du droit, une autre difficulté surgit quand il s'agit de définir le droit lui-même. Droit objectif (comme dans

G. Brausch (dir.), Bruxelles, Paris, Bruylant, LGDJ, 2002, p. 43 ; voy. aussi N. RouLAND, L'Anthropologie juridique, Paris, PUF, 1990, p. 46 et s.

${ }^{44}$ Ibidem ; Synthétisant les travaux récents de l'anthropologie juridique française, Rodolfo SACco propose une définition qui assimile droit et règle : " II a été proposé d'appeler droit la règle disposition générale et permanente - et de considérer la juridicité comme une qualité propre non seulement au droit mais également à tout mécanisme de contrôle assumé par un groupe social vis-à-vis des comportements de ses membres, le droit ayant recours aux sanctions, et les autres mécanismes ayant pour but de retrouver l'harmonie sociale si celle-ci a été rompue " (R. SAcco, Anthropologie juridique. Apport à une macro-histoire du droit, Paris, Dalloz, 2008, p. 9). Cette définition ne laisse toutefois pas de place à la relation entre deux individus, ni au commandement qui épuise ses effets par sa première application. Faut-il ainsi considérer qu'un acte individuel de l'administration ne contient pas de règle de droit ? De plus, la distinction opérée entre droit et juridicité d'une part, et entre sanction et autres mécanismes d'autre part, soulève d'innombrables questions qu'il ne convient pas d'examiner ici en profondeur. Rodolfo SAcco souligne en particulier C. EBERHARD et G. VeRnicos (éds.), La Quête anthropologique du droit. Autour de la démarche d'Étienne Le Roy, Paris, Karthala, 2006.

${ }^{45}$ L. AsSIER-ANDRIEU, Le droit dans les sociétés humaines, Paris, Nathan, 1996, p. 44-45.

${ }^{46}$ Ibidem, p. 45-47 ; É. DURKHEIM, De la division du travail social, Paris, Quadrige/PUF, $2^{\mathrm{e}}$ éd., 1991, p. 108-109.

${ }^{47}$ H.L.A. HART, Le concept de droit, Bruxelles, Facultés universitaires Saint-Louis, 1976, p. 14 ; L. ASSIER-ANDRIEU, op. cit., p. 44-45.

${ }^{48}$ Norbert RoULAND, quant à lui, considère que « si l'État et le droit peuvent former un couple, celui-ci n'est pas indissociable ". L'État résulterait selon lui du choix des sociétés "d'étendre la part du droit et de l'expliciter en normes, codifiées ou non (...) » (N. ROULAND, L'Anthropologie juridique, Paris, PUF, 1990, p. 48). L'État serait donc l'expression de la place plus large accordée au droit dans une société. Le critère de basculement d'une société sans État à une société à État demeure néanmoins flou, de même que la différence entre norme et droit.

49 Ibidem, p. 68.

${ }^{50}$ Cette saveur en plus est précisée : «D'abord, spécifiquement juridique est la norme qui devra prévaloir parmi d'autres registres normatifs. Ensuite, la fonction juridique tire des tumultes qui la font intervenir un "caractère d'officialité" sous la foi duquel se résout la friction » (L. ASSIERANDRIEU, présentation de K. N. LleWEllyn et E. AdAmson Hoebel, La Voie Cheyenne. Conflit et jurisprudence dans la science primitive du droit, Bruxelles, Paris, Bruylant, LGDJ, 1999, p. XI). 
droit français), droit subjectif (comme dans droits de l'homme), objet de discipline académique (comme dans études de droit), etc. : les usages du mot sont nombreux et les réalités recouvertes, souvent floues.

$\mathrm{Au}$ fond, le problème du droit dans les sociétés dites primitives est essentiellement un problème de définition. Avec Robert Jacob, on relève à cet égard qu'« il a manqué aux sciences juridiques du XXe siècle l'équivalent de ce qu'a été la révolution saussurienne dans les sciences du langage. À chaque philologie, dont l'objet spécial est une langue ou un groupe de langues, est venue se superposer la linguistique, science générique de la communication par le verbe. Les savoirs philologiques correspondent à la variété des aires culturelles, tandis que la linguistique tend à recomposer l'unité de l'homme. Mais, on le sait, la genèse de la linguistique générale n'a été rendue possible que par la création d'un corps de concepts propres, assez précis et assez plastique pour s'adapter à l'analyse de tout langage et, pour cette raison, radicalement arraché à l'horizon où chaque grammaire particulière enfermait le philologue. Faute d'avoir entrepris pareil effort, les sciences historiques et comparatives du droit en sont encore à procéder par tâtonnements, un peu comme si l'on persistait à ne comprendre les langues amérindiennes ou bantoues qu'à travers ce qui les rapprocherait confusément de l'indo-européen ${ }^{51}$. Ce qui manque à la science juridique est donc une méthode d'appréhension réellement universelle de son objet. Le terme droit est tellement galvaudé et imprécis que l'étude du phénomène qu'il est censé recouvrir jusque dans les sociétés "primitives " ne peut se passer d'une réflexion préalable sur sa définition même.

Afin de mieux appréhender ce que recouvre le phénomène juridique, nous laisserons ainsi provisoirement de côté, pour les besoins de la démonstration, le mot droit, incurablement ambigu, en nous fondant sur la méthode microscopique proposée par le théoricien belge du droit Lucien François $^{52}$. Si, en plus de l'ambiguïté dont souffre le mot, l'on se rappelle que « droit (ius, Recht, law, diritto, pravo) est une notion qui n'a pas d'équivalent précis dans toutes les cultures $»^{53}$, la nécessité de chercher une autre grille d'analyse se fait encore plus criante: notre but n'est pas d'appliquer artificiellement nos catégories à des cultures qui ne les connaissent pas telles quelles mais de chercher à comprendre un phénomène. Lucien François offre une méthode progressive qui, en partant de l'unité de base d'un phénomène social donné (que le lecteur occidental aura un peu trop vite tendance à

${ }^{51}$ R. JACOB, « Le droit, l'anthropologue et le microscope », op. cit., p. 44.

52 L. FRANÇOIS, Le Cap des Tempêtes. Essai de microscopie du droit, Bruxelles-Paris, BruylantLGDJ, 2001, $2^{\mathrm{e}}$ éd., 2012.

${ }^{53}$ R. SACCO, op. cit., p. 8. 
qualifier de " juridique ", au risque de manquer ce qui fait l'un des principaux apports de la démarche microscopique), se veut réellement universelle. Ainsi, l'application des outils conceptuels élaborés par Lucien François aux sociétés étudiées par Clastres permettra d'en apprécier la pertinence et, nous l'espérons, de remettre en cause les conceptions évolutionnistes encore trop souvent présentes dans le champ de l'anthropologie juridique pour mettre en lumière non ce qui nous sépare mais bien ce qui nous unit ${ }^{54}$. Pierre Clastres comme Lucien François voient dans la relation de pouvoir élémentaire l'embryon du phénomène étatique. Cette convergence de vues entre deux auteurs dont les travaux semblent, a priori, poursuivre des voies distinctes

\footnotetext{
${ }^{54}$ Du reste, la question de la coexistence de plusieurs « ordres juridiques » est au fondement de la théorie microscopique du droit (Lucien FRANÇOIS est d'ailleurs, avec Pierre GOTHOT, l'un des deux traducteurs en langue française de L'Ordinamento giuridico de Santi Romano, à la base de la théorie dite " de la pluralité des ordres juridiques » : L'Ordre juridique, Paris, Dalloz, 2002). Au début du Cap des Tempêtes, Lucien FRANÇOIS pose le constat suivant : " Nous lisons, sous la plume de juristes ou de sociologues, qu'il existe - au sein de sectes, d'entreprises, de syndicats, de partis, de mouvements révolutionnaires, de sociétés secrètes, de gangs, de milieux d'affaires et jusque dans "le milieu" - bon nombre de "règles sociales, sinon juridiques", de "règles infrajuridiques", de "règles comparables à des règles de droit". Or de telles expressions parlent deux fois du concept de droit puisqu'elles soulignent la proximité de certains phénomènes par rapport au droit mais impliquent qu'ils n'en font pas partie. Elles sont donc à leur tour autant d'allusions vagues à une frontière supposée du droit et du non-droit, frontière au-delà de laquelle se trouveraient certaines règles alors que les règles étatiques et canoniques (car l'expression "droit canonique" reste couramment employée) se tiendraient en deçà » (L. FRANÇOIS, Le Cap des Tempêtes..., op. cit., p. 8). Lucien FRANÇOIS cherche des outils rigoureux capables de faire droit à la spécificité de chacun de ces phénomènes. II ne s'agit pas de dire ce qui est et ce qui n'est pas mais, au contraire, de partir de ce qui est et de saisir la manière dont ces phénomènes existent ensemble. À cet égard, les outils du juriste belge pourraient s'avérer particulièrement précieux pour penser le pluralisme juridique. Dans le champ de l'anthropologie juridique, la question est abondamment traitée. Avec Rodolfo SACCO, on signale les travaux de G. GURVITCH, L'Expérience juridique et la philosophie pluraliste du droit, Paris, Pédone, 1935 ; J. GILISSEN (dir.), Le Pluralisme juridique, Bruxelles, Éditions de I'Université de Bruxelles, 1972 ; J.-G. BELLEY, Conflit social et pluralisme juridique en sociologie du droit, Thèse, Paris II, 1977 ; J. GRIFFITHS, "Anthropology of Law in the Netherlands in the 1970 », Nieuwsbrief voor Nederlandstalige Rechtssociologen, Rechtsantropologen en Rechtspsychologen, 1983, p. 132 ; M. CHIBA (éd.), Asian Indegenous Law in Interaction with received Law, Londres, New York, KPI Publishers, 1986 ; F. VON BENDA-BECKMANN et F. STRIJBOSCH (éds.), Anthropology of Law in the Netherlands, Dordrecht, Foris, 1986 ; R. MACDONALD, « Normativité, pluralisme et sociétés démocratiques avancées », Rev. de droit de l'Univ. de Sherbrooke, 2002, p. 133 ; R. MOTTA, "Maîtres chez eux". Sovranità domestica e diritti ancestrali delle prime nazioni in Nuova Francia e Canada », Materiali per una storia della cultura giuridica in Italia, 2001, n¹, p. 211 ; N. ROULAND, S. PIERRÉCAPS et J. POUMARĖDE, Droit des minorités et des peuples autochtones, Paris, PUF, 1996 ; A. LAJOIE, R.A. MACDONALD, R. JANDA et G. ROCHER (éds.), Théories et émergence du droit : pluralisme, surdétermination, effectivité, Montréal/Bruxelles, éd. Thémis, Bruylant, 1998; A. GAGNON et F. Rocher (éds.), The Conditions of Diversity in Multinational Democracies, Montréal, Canada IRPP - Mc Gill University Press, 2003. Voy. aussi les périodiques : Journal of Legal Pluralism ; Law and Anthropology ; Newsletters of the Commission on Folk-Law and Legal Pluralism.
} 
rend leur confrontation sinon indispensable, du moins particulièrement stimulante.

Une fois isolée, grâce à cette méthode, la particule élémentaire du phénomène qualifié, dans la pensée occidentale, de " juridique " (que nous appellerons donc systématiquement, dans la suite, " phénomène juridique ", en priant le lecteur de garder constamment à l'esprit le caractère ethnocentré de cette expression), nous démontrerons que les sociétés sans État ne sont pas pour autant des sociétés a-juridiques.

\section{B. L'approche microscopique du phénomène juridique}

Pour surmonter les ambigüités du concept de droit, Lucien François propose de recourir à un ensemble de néologismes, précisément définis et désignant autant de stades de complexification au départ d'un phénomène embryonnaire ('injonction du brigand « La bourse ou la vie ! ») pour atteindre la structure élaborée de ce que l'on appelle, dans le langage commun, "État ». II élabore une méthode et met au point des instruments d'analyse permettant, selon lui, d'appréhender la multiplicité des manifestations du phénomène juridique, sans tomber dans les ornières de l'ambivalence sémantique : une révolution saussurienne de la science juridique, en quelque sorte. Nous nous contenterons d'examiner les éléments pertinents de sa théorie pour l'analyse du fonctionnement juridique des sociétés étudiées par Clastres.

\section{Le jurème}

La figure la plus élémentaire du phénomène étudié par Lucien François est désignée par le néologisme jurème, défini comme " toute apparence, produite par un humain, du vœu d'obtenir une conduite humaine, apparence de vœu munie d'un dispositif tel que la résistance d'un des destinataires déclenche une pression en sens contraire par menace de sanction $»^{55}$. Cinq éléments concourent donc à la survenance d'une telle situation :

a) le pouvoir d'exercer une pression par menace de désagrément. II importe peu que le pouvoir d'infliger le désagrément soit réel : il suffit de faire croire qu'on sera en mesure d'infliger la sanction ou de la faire infliger (on pourrait par exemple se servir d'un revolver factice, ou encore s'appuyer sur les rigueurs d'une divinité ou de quelque puissance maléfique, pour menacer l'être humain dont on exige un comportement déterminé) ;

\footnotetext{
${ }^{55}$ L. FRAnçoIs, Le Cap des Tempêtes..., op. cit., p. 42.
} 
b) le destinataire doit être conscient, sensible et exposé. " II faut donc, pour pouvoir émettre un jurème, avoir devant soi des êtres à la fois capables de se savoir menacés et sensibles à ce dont on les menace $»^{56}$. II est nécessaire, en outre, que le destinataire du jurème ne soit pas en mesure d'échapper au pouvoir qui le menace $^{57}$;

c) le dispositif de pression doit être de nature à combattre la résistance à un vœu. Ainsi, la violence gratuite ne participe pas à la formation d'un jurème ;

d) le vœu doit être perçu comme impératif ${ }^{58}$ et, partant, catégorique, " c'est-à-dire comme indépendant de ce que le destinataire désire $»^{59}$;

e) le vœu et la menace doivent être évidents ou, à défaut, notifiés.

Ces cinq éléments sont déjà réunis dans la brève rencontre au cours de laquelle un brigand enjoint à un passant de lui remettre son argent sous la menace d'une arme. Mais ils sont susceptibles d'entrer dans la composition de formes beaucoup plus complexes de rapports de pouvoir - jusqu'à celle, extrêmement sophistiquée, de l'État. Celui-ci est caractérisé, pour Lucien François, par un type particulier de jurème : le jurème de suprématie. II s'agit "d'interdire en un lieu donné toute violence physique majeure non autorisée ${ }^{60}$.

\section{Complexification des structures de pouvoir, cercle d'exigence} mutuelle et homodoxie

Le simple jurème ne suffit pas à décrire le fonctionnement des rapports de pouvoirs au sein d'un groupe social. Un même individu peut émettre plusieurs jurèmes liés les uns aux autres de manière cohérente : ils sont tantôt préparatoires, tantôt modificatifs et sont créés grâce à " un même " pouvoir, à « une même » capacité de pression par menace. Ce plus petit système de

\footnotetext{
56 Ibidem, p. 53.

${ }^{57}$ Ibidem, p. 53-54.

58 "Le jurème n'est donc souvent qu'un vœu accompagné d'un dispositif de pression dormant, provisoirement inerte. La pression n'est alors que virtuelle. Mais même lorsque le dispositif n'est pas en mouvement, l'auteur le rend présent en se montrant résolu à contraindre au cas où le vœu rencontrerait une résistance. J'appellerai impérativité l'apparence ainsi créée, essentielle au jurème » (ibidem, p. 59).

59 Ibidem.

60 Ibidem, p. 251.
} 
pouvoir sous la coupe d'un même individu (le "maître ») est appelé $\operatorname{archème~}^{61}$.

Lorsque plusieurs individus, chacun maître d'un archème, acceptent de collaborer pour former une structure de pouvoir au sein d'un groupe social, leurs archèmes s'agglutinent : chaque individu « fait savoir qu'il coopère avec les autres et que les messages jurémiques de ceux-ci sont relevants pour lui ${ }^{62}$. Autrement dit, chaque individu fait siens - d'une certaine manière ces messages jurémiques. II en résulte plus qu'une simple addition d'archèmes : si $A$ prend en compte les exigences de $B, C$ ne peut désobéir à $B$ sans déplaire à $A$ et réciproquement. Les exigences de l'un deviennent celles de l'autres. Cette agglutination forme ce que Lucien François appelle des agrégats ${ }^{63}$. On distingue deux types d'agrégats. D'une part, les agrégats formés par agglutination d'archèmes sont symétriques lorsqu'ils sont formés d'archèmes se situant au même niveau, d'archèmes non hiérarchisés entre eux. C'est le cas lorsque, dans une file d'attente, chacun des individus coalisés qui la compose fait connaître sa désapprobation à une personne tentant d'en dépasser une autre dans la file. D'autre part, les agrégats peuvent être dans un rapport hiérarchique ; ils sont alors polarisés. C'est le cas, par exemple, au sein d'une organisation criminelle où s'agglutinent des agrégats à la tête desquels se trouve l'archème du chef.

La cohésion d'un groupe social est par ailleurs assurée par une certaine homogénéité des convictions normatives. Certains principes sont, en effet, tenus pour essentiels par tous, si bien que chacun attend des autres qu'il les respecte. Cette homogénéité de convictions est appelée homodoxie ${ }^{64}$. Puisque chacun attend des autres qu'ils respectent ces exigences, plusieurs agrégats symétriques se superposent dans lesquels chacun est à tour de rôle le destinataire des messages jurémiques coalisés des autres. À la différence d'agrégats issus de l'agglutination d'archèmes où la structure de pouvoir (ceux qui commandent) dirige ses exigences vers l'extérieur (ceux qui doivent obéir), la superposition d'archèmes dans cette configuration forme un cercle d'exigence mutuelle dont les membres commandent et doivent obéir tout à la fois ${ }^{65}$. "Chaque membre du groupe est à la fois destinataire dans un de ces

\footnotetext{
${ }^{61}$ Ibidem, p. 129 et s.

62 Ibidem, p. 232.

${ }^{63}$ Ibidem, p. 195-199.

64 Ibidem, p. 207-212; sur l'homodoxie des sociétés " primitives », voy. en particulier ibidem, p. 211-212.

65 Ibidem, p. 199-202.
} 
agrégats et maître d'un archème entrant dans la composition de tous les autres $»^{66}$.

\section{Le nimbe}

Le nimbe vise à renforcer l'efficacité des messages jurémiques via un habillage adéquat de la notification du vœu et de la menace de sanction en cas de désobéissance. Ce mécanisme, dont le nom renvoie à la technique iconographique qui consiste à envelopper d'un cercle lumineux certains personnages en vue de les magnifier, vise tantôt à renforcer l'apparence menaçante de l'émetteur du jurème, tantôt à atténuer le désagrément lié à la contrainte en présentant le jurème ou son auteur sous des atours séduisants ou de nature à susciter l'adhésion du destinataire. Ce maquillage orienté de la réalité se manifeste essentiellement, mais pas uniquement, dans un certain usage du langage.

Ainsi, les travaux préparatoires des textes législatifs, la motivation des actes administratifs, la propagande qui accompagne certaines mesures gouvernementales et autres discours justificatifs relèvent du nimbe. Ils visent à justifier, à présenter avantageusement, une mesure potentiellement désagréable. L'usage récurrent d'euphémismes dans les textes légaux constitue aussi une forme de nimbe ${ }^{67}$. Dans les sociétés " archaïques » en particulier, " un autre euphémisme (...) consiste à appeler dons, cadeaux, libéralités - comme s'ils étaient un pur effet de la générosité - des présents qu'un usage impose dans certaines circonstances (mariage, etc.) sous peine d'être déconsidéré et traité en conséquence, mais dont il est requis de parler comme s'ils n'étaient pas obligatoires ${ }^{68}$.

Selon Clastres, ce don obligatoire, cette générosité nimbée, caractérise les rapports des Indiens avec leur chef. Derrière ces échanges se camoufle une rationalité plus profonde enfouie sous une double couche de nimbe : le don est une technique de maîtrise du pouvoir.

Le chef est chargé de maintenir la paix. En fait, il a intérêt au maintien de la paix : "le leader ne possède aucun pouvoir décisoire ; il n'est jamais assuré que ses "ordres" seront exécutés: cette fragilité permanente d'un pouvoir sans cesse contesté donne sa tonalité à l'exercice de la fonction : le pouvoir du chef dépend du bon vouloir du groupe. On comprend dès lors l'intérêt direct du chef à maintenir la paix : l'irruption d'une crise destructrice de l'harmonie interne appelle l'intervention du pouvoir, mais suscite en même

${ }^{66}$ Ibidem, p. 200.

${ }^{67}$ Ibidem, p. 91.

68 Ibidem, p. 91-92. 
temps cette intention de contestation que le chef n'a pas les moyens de surmonter ${ }^{69}$. À cette mission première se greffent trois rapports d'obligations qui, faute de réciprocité, doivent certes s'analyser comme des dons mais des dons au service d'un objectif politique ou, si l'on veut, antipolitique : il s'agit de l'obligation du chef de parler et d'être généreux en biens et de l'obligation du groupe de donner des femmes au chef, lequel a le privilège de la polygynie. La circulation et l'échange de ces trois éléments (paroles, biens, femmes) caractérise le passage de la nature à la culture. En agissant sur cet échange, les Indiens agissent sur la structuration même du groupe comme société. Or, « il est évident que pour le groupe, qui s'est dessaisi au profit du chef d'une quantité importante de ses valeurs les plus essentielles - les femmes -, les harangues quotidiennes et les maigres biens économiques dont peut disposer le leader ne constituent pas une compensation équivalente ${ }^{70}$. Ce qui semblait relever d'une logique d'échange est bien un don, faute de réciprocité conséquente. D'ailleurs, le pouvoir est héréditaire, il ne circule pas ; le pouvoir n'échoit pas au plus riche ni au meilleur orateur. Le refus de l'échange et, partant, l'absence de réciprocité isole chaque signe dans un circuit propre: « le pouvoir entretient un rapport privilégié aux éléments dont le mouvement réciproque fonde la structure même de la société ; mais cette relation, en leur déniant une valeur qui est d'échange au niveau du groupe, instaure la sphère politique non seulement comme extérieure à la structure du groupe, mais bien plus comme négatrice de celle-ci : le pouvoir est contre le groupe, et le refus de la réciprocité, comme dimension ontologique de la société, est le refus de la société elle-même ${ }^{71}$. En situant la parole hors du groupe et hors de la sphère des échanges - et donc de la communication -, le risque de voir émerger un discours prétendant à la coercition est écarté car « il est si peu nécessaire au discours du chef d'être écouté que les Indiens ne lui prêtent souvent aucune attention ${ }^{72}$. Un chef qui tenterait de rendre son pouvoir effectif se verrait abandonné par le groupe. Les femmes sont dès lors plus un objet de chantage contre le chef qu'un réel privilège de celui-ci.

Le phénomène de nimbe apparaît donc double dans l'institution de la chefferie : d'une part, derrière des échanges apparents se cachent en réalité des flux à sens unique et, d'autre part, ces flux isolés permettent au groupe de maîtriser le chef et les flux de biens, de paroles et de femmes, lesquels permettraient - selon Clastres - de faire société (politique ?) si leur communication était libérée.

\footnotetext{
${ }^{69}$ P. CLASTRES, La Société contre l'État, op. cit., p. 34.

${ }^{70} \mathrm{Ibidem}$, p. 35.

${ }^{71}$ Ibidem, p. 38.

72 Ibidem, p. 41.
} 


\section{Le fonctionnement juridique des sociétés dites primitives à la lumière de l'approche microscopique}

1. Impératif d'égalité et initiation : empêcher l'émergence d'un pouvoir effectif centralisé

Nous avons déjà mentionné, à plusieurs reprises, l'impératif d'égalité qui s'impose aux membres des sociétés "primitives " et insisté, avec Clastres, sur la structure même de ces sociétés, qui empêche l'émergence d'un pouvoir effectif centralisé par le maintien d'une stricte égalité. Cette exigence s'exprime particulièrement à travers des rites initiatiques que Clastres n'hésite pas à comparer à de la torture. II s'agit, une fois de plus, d'empêcher la prise de pouvoir par un individu. En s'assurant que la société forme une entité parfaitement homogène, les chances de voir émerger un rapport de pouvoir centralisé et, par voie de conséquence, un rapport de domination au sein du groupe sont encore réduites. En effet, il n'y a de droit que dans un rapport intersubjectif ${ }^{73}$. II semblerait que la société primitive cherche à se constituer comme sujet unique, de sorte que la tentative de prise de pouvoir ne pourrait venir que de l'extérieur. Elle serait vite neutralisée puisque, on l'a vu également, les sociétés dites primitives possèdent un lieu du pouvoir, certes ineffectif, mais néanmoins opposable. De même, la loi venue de l'extérieur serait immédiatement neutralisée par la loi interne, la loi de la société.

\section{Examinons l'extrait suivant :}

Dans la mesure exacte où l'initiation est, indéniablement, mise à l'épreuve du courage personnel, celui-ci s'exprime, si l'on peut dire, dans le silence opposé à la souffrance. Mais, après l'initiation, et toute souffrance oubliée déjà, subsiste un surplus, un surplus irrévocable, les traces que laisse sur le corps l'opération du couteau ou de la pierre, les cicatrices des blessures reçues. Un homme initié, c'est un homme marqué. Le but de l'initiation, en son moment tortionnaire, c'est de marquer le corps : dans le rituel initiatique, la société imprime sa marque sur le corps des jeunes gens. Or, une cicatrice, une trace, une marque sont ineffaçables. Inscrites dans la profondeur de la peau, elles attesteront toujours, éternelles, que si la douleur peut n'être qu'un mauvais souvenir, elle fut néanmoins éprouvée dans la crainte et le tremblement. La marque est un obstacle à l'oubli, le corps lui-même porte imprimées sur soi les traces du souvenir, le corps est une mémoire. (...) Le rituel initiatique est une pédagogie qui va du groupe à l'individu, de la tribu aux jeunes gens. Pédagogie d'affirmation et non de dialogue : ainsi les initiés doivent-ils rester silencieux

\footnotetext{
${ }^{73}$ L. FRANÇOIS, Le Cap des Tempêtes..., op. cit., p. 66.
} 
sous la torture. Qui ne dit mot consent. À quoi consentent les jeunes gens ? Ils consentent à s'accepter pour ce qu'ils sont désormais : des membres à part entière de la communauté. Rien de moins, rien de plus. Et ils sont irréversiblement marqués comme tels. Voilà donc le secret que dans l'initiation le groupe révèle aux jeunes gens : "Vous êtes des nôtres. Chacun de vous est semblable à nous, chacun de vous est semblable aux autres. Vous portez même nom et n'en changerez pas. Chacun de vous occupe parmi nous même espace et même lieu : vous les conserverez. Aucun de vous n'est moins que nous, aucun de vous n'est plus que nous. Et vous ne pourrez pas l'oublier. Sans cesse, les mêmes marques que nous avons laissées sur votre corps vous le rappelleront » (...). La société dicte sa loi à ses membres, elle inscrit le texte de la loi sur la surface des corps. Car la loi qui fonde la vie sociale de la tribu, nul n'est censé l'oublier ${ }^{74}$.

L'initiation inscrit littéralement la loi de la société sur le corps de ses membres (tatouages, scarifications...). Tous les membres de la tribu sont marqués par la même loi fondamentale: "Tu ne vaux pas moins qu'un autre, tu ne vaux pas plus qu'un autre ${ }^{75}$. Cette inscription viendra sans cesse se rappeler à l'individu. Le mécanisme est d'une subtilité étonnante pour des sociétés qualifiées de "primitives ». La société semble consciente du double foyer de la menace de domination : la société elle-même par les individus qui la composent, d'une part, et, d'autre part, l'extérieur. Pour empêcher la loi de l'extérieur, il faut une loi de l'intérieur, une loi considérée comme plus fondamentale que la société opposera et fera prévaloir sur toute autre norme. II ne faut pas non plus que cette loi puisse être renversée de l'intérieur par un individu ou un groupe d'individus par trop ambitieux et désireux d'accaparer le pouvoir au sein du groupe. Ce n'est alors pas sur le mécanisme même de la loi que la société joue mais sur son contenu et sur la manière de la communiquer (de la notifier, pour reprendre le vocabulaire de Lucien François). Le contenu de la loi, c'est l'interdiction de l'inégalité, c'est l'interdiction du rapport de pouvoir. Le mode de notification de la loi aux membres de la tribu empêche qu'ils ne l'oublient, qu'ils ne sentent plus l'exposition jurémique première : « les cicatrices dessinées sur le corps, c'est le texte inscrit de la loi primitive, c'est, en ce sens, une écriture sur le corps ${ }^{76}$. Au sein du corps social, les seuls ailleurs possibles desquels pourrait émaner le phénomène juridique répugnent à l'inégalité, ils ne la conçoivent même pas ou, du moins, ils ne l'envisagent pas car les individus ne sont pas Un, mais bien inscrits dans une multiplicité qui les rassemble. Cette loi vise certes à

\footnotetext{
${ }^{74}$ P. CLASTRES, La Société contre l'État, op. cit., p. 156-157.

${ }^{75}$ Ibidem, p. 158.

${ }^{76}$ Ibidem, p. 159.
} 
empêcher l'émergence interne de l'inégalité, mais elle vise aussi à conjurer la loi externe, la loi séparée : " c'est pour conjurer cette loi-là, loi fondatrice et garante de l'inégalité, c'est contre la loi d'État que se pose la loi primitive ${ }^{77}$. L'homogénéisation du corps social par le marquage des corps individuels que réalise l'initiation empêche, en dernière instance, l'émergence du droit dans son aspect irréductiblement relationnel (un être humain commande à un autre, obligé d'obéir). II n'y a de rapport de pouvoir, en particulier il n'y a de situation jurémique, qu'entre deux individus au moins. Si le sujet primitif est parfaitement homogène, s'il n'est pas séparé des autres mais forme avec eux un tout indivisible, si l'homodoxie est totale, le rapport de pouvoir semble alors impossible et, partant, l'émergence de l'État et de son droit.

2. Cercle d'exigence mutuelle: assurer un pouvoir diffus par lequel chacun commande et obéit aux autres

Cette homogénéité connaît toutefois des ratés: on observe des infractions à la loi des Aché. Ces dissidences proviennent, selon Clastres, du contact avec les Blancs, avec l'extérieur. Pour déceler une dimension jurémique dans l'organisation du groupe, il faut, nous l'avons vu, qu'un dispositif de sanction puisse être mis en œuvre. Or la figure du cercle d'exigence mutuelle permet de concilier impératif d'égalité et émission de messages jurémiques. Les sociétés sans État ne se maintiennent pas par une homogénéité parfaite du Nous mais par une disposition particulière des injonctions jurémiques.

Les Guyaki pratiquent l'initiation, fragmentant la vie des individus et structurant la société, reproduction ritualisée de la mythologie des Indiens. " On ne peut pas être à la fois enfant et adulte, kybuchu et séducteur de femmes, c'est l'un ou l'autre, l'un après l'autre, d'abord on est Pénis, ensuite Labret: on ne doit pas laisser les choses se confondre, les vivants ici, les morts là-bas, les enfants d'un côté, les initiés de l'autre ${ }^{78}$. Après leurs premières règles, les filles doivent subir un rituel initiatique très douloureux visant à les purifier. Avant le contact avec les Blancs, les jeunes indiens ne s'opposèrent jamais à ces rites pourtant douloureux. Chachugi, elle, résista. " "Pas de scarifications! La fille manque totalement de courage". Pour la première fois, la loi des Aché n'est pas respectée. Chachugi a eu peur, elle n'a pas voulu affronter le jaycha bowo, l'épreuve de la douleur l'a terrifiée. Comment cela est-il possible ? Les gens sont furieux. Mais que peuvent-ils faire ? Chacun sait bien que si le "sang était sorti" quelques mois plus tôt, avant le contact avec les Blancs, pas un instant Chachugi n'aurait même

77 Ibidem

${ }^{78}$ Ibidem, p. 137. 
songé à se dérober à ce qui de tout temps fut le devoir des femmes Aché. (...) Chachugi a craint de devenir une grande personne, une Aché véritable, elle a voulu rester "femme nouvelle". Pareille chose pouvait-elle durer ? Toujours est-il qu'un matin froid de juin on découvrit, déjà raidi, le cadavre de la petite dare pou; elle était morte pendant la nuit, en silence. On ne commenta point. Ache rö kwaty, ce sont les Aché qui savent ${ }^{79}$.

Dans le cas de Chachugi, la sanction n'était sans doute pas clairement connue d'avance. Elle savait cependant qu'en refusant de se soumettre au rituel d'initiation, elle se plaçait hors de la société. Sa mise à mort par le groupe n'est-elle finalement pas la mise en œuvre la plus aboutie de cette exclusion?

L'obligation de se soumettre à l'initiation et, partant, d'assurer la perpétuation des lois ancestrales s'analyse bien en une obligation jurémique : premièrement, le pouvoir d'exercer une pression par menace de désagrément est détenu par l'ensemble du groupe à l'exception de Chachugi, la menace de désagrément étant l'exclusion du groupe par un moyen quelconque. Deuxièmement, Chachugi est consciente et sensible à cette menace : elle sait que personne ne peut faire partie intégrante du groupe sans subir l'initiation. Elle n'a pas pris la fuite et est, dès lors, restée exposée à la menace. Troisièmement, le dispositif de pression mis en place par le groupe est de nature à combattre la résistance au vœu qu'il émet : Chachugi n'aurait pas été assassinée si elle avait accepté de subir l'initiation. Quatrièmement, l'initiation est imposée par le groupe à la jeune indienne indépendamment de ce qu'elle désire et, cinquièmement, le vœu et la menace étaient évidents pour Chachugi ; les nombreuses initiations auxquelles elle a assisté suffisent à lui faire comprendre ce qu'on attend d'elle.

À certaines règles, dont la transgression est certainement plus concevable, sont associées des sanctions plus précises. Par exemple, chez les garçons, l'initiation se déroule en deux phases. La première phase est l'imbi mutu. Lorsque les garçons ne peuvent plus réfréner leurs pulsions sexuelles, ils demandent « la perforation dans la lèvre, afin d'être un chasseur confirmé et de pouvoir arborer désormais le labret qui, marquant [leur] entrée dans l'âge adulte, [leur] ouvrirait sans restriction l'accès aux femmes convoitées depuis longtemps $»^{80}$. Tant que la plaie n'est pas cicatrisée, tant que le kaimbo, la ficelle nouée à l'intérieur de la lèvre qui empêche le trou de se refermer, ne casse pas, les jeunes initiés ne peuvent se laisser aller à leurs désirs charnels. "Sinon, il arrive le pire malheur pour un homme, la

${ }^{79}$ Ibidem, p. 152-153.

80 Ibidem, p. 129. 
malchance à la chasse $»^{81}$. Que cette sanction soit fondée sur une superstition et qu'elle provienne de l'extérieur du groupe importe peu.

Ces exemples prouvent qu'il existe des phénomènes jurémiques (c'està-dire juridiques) au sein des sociétés sans État : celles-ci produisent bien des jurèmes. L'impératif d'égalité et la maîtrise structurale du pouvoir empêchent cependant que ces jurèmes émanent d'individus isolés au détriment des autres. Le phénomène juridique ne se déploie que dans la figure du cercle d'exigence mutuelle où chacun est à la fois émetteur et destinataire d'obligations jurémiques et garant de leur respect. Chachugi n'a pas été assassinée par un membre trop zélé du groupe, il ne s'est trouvé personne pour la défendre. Le silence entendu des Indiens est éloquent.

\section{$D$. Synthèse}

La société dite primitive vise à empêcher l'État. Elle se structure pour cela de manière à empêcher l'émergence d'une relation de domination - autre que celle de tous sur chacun - et donc, le phénomène embryonnaire du droit, plus particulièrement du droit étatique. Empêcher l'État, c'est empêcher le droit étatique mais par le droit du groupe social homogène ; empêcher l'État, c'est, dans les termes de Lucien François, empêcher la formation de jurèmes hors du cercle d'exigence mutuelle et grâce à celui-ci. La société " primitive " n'est pas a-juridique. Elle est structurée par un ensemble de règles assorties d'une menace de sanction. Ces règles sont l'expression d'une loi ancestrale dont le contenu est connu de tous et sans cesse rappelé par un chef sans pouvoir dont le devoir est de parler.

"Le lieu du pouvoir, c'est la société ${ }^{82}$. Cette formule traduit plus simplement la figure du cercle d'exigence mutuelle. Chaque Indien, maître d'un archème, émet un ensemble d'exigences de même contenu vis-à-vis des autres. Ces archèmes homonomes s'agglutinent, formant ainsi des agrégats symétriques. Chacun exige dès lors de tous les autres qu'ils respectent la loi des ancêtres et chacun sait que tous les autres attendent de lui qu'il fasse de même. Ce cercle d'exigence mutuelle constitue la structure dans laquelle s'inscrit le juridique chez les «Sauvages ».

L'interdiction de l'inégalité empêche cette configuration de changer. Le droit ne peut se manifester dans les sociétés dites primitives que dans une symétrie parfaite. Comment en effet inclure un jurème émanant d'un individu et sans réciprocité dans un cercle d'exigence mutuelle? C'est impossible. Inconsciemment, les Aché l'ont bien compris. Le contenu de la norme

81 Ibidem, p. 140.

82 Ibidem, p. 176. 
fondamentale empêche l'émergence d'une dissymétrie dans les rapports sociaux. En revenant au mot droit et en jouant sur son ambigüité, on peut écrire que l'impératif d'égalité empêche le droit ${ }^{83}$ par le droit ${ }^{84}$ et que le droit ${ }^{85}$, en empêchant le droit ${ }^{86}$, empêche l'État.

\section{Des sociétés sans liberté ?}

II est clair, à ce stade, que l'égalité - ou du moins, l'égalité forcée au moyen de pressions réciproques - est la condition la plus basique et la plus fondamentale de la non-apparition de l'État. Le droit ne s'exprime en effet, dans les sociétés dites primitives, que par la figure du cercle d'exigence mutuelle. Cette configuration implique une parfaite symétrie des rapports. L'impératif d'égalité, dans la mesure où il est obéi, empêche toute dissymétrie. La rupture de l'égalité entraînerait la fin du cercle ou, du moins, l'émission de messages jurémiques hors de ce cercle. Un rapport de domination s'installerait alors, rendant possible l'émission de nouveaux jurèmes, y compris le jurème de suprématie propre à l'État. L'émission d'un jurème de suprématie ne peut en effet avoir lieu au sein d'un cercle d'exigence mutuelle, a fortiori lorsque celui-ci est marqué par des agrégats symétriques et une homodoxie donnée, puisqu'il suppose comme destinataire potentiel toute personne se trouvant sur un certain territoire, que cette personne soit membre du cercle ou non. En d'autres termes, le champ d'application du cercle d'exigence mutuelle est d'ordre personnel, alors que le champ d'application du jurème de suprématie est d'ordre territorial.

Le pouvoir politique, fondé sur le droit étatique et donc sur un pouvoir de contrainte, est par nature inégalitaire. On sait désormais que l'inscription de la loi dans le corps social et dans le corps des individus prévient l'émergence d'une loi venue de l'extérieur, qu'elle garantit l'indépendance de la tribu, qu'elle garantit, pour le formuler autrement, sa liberté. Du point de vue de la société, prise comme un tout, il est certain que l'égalité est le fondement de sa liberté. Si l'on se place d'un point de vue individuel, en revanche, la question s'avère plus délicate.

\footnotetext{
${ }^{83}$ C'est-à-dire les jurèmes émanant unilatéralement d'un (ou plusieurs) émetteur(s) à l'égard d'un (ou plusieurs) destinataire(s).

${ }^{84}$ C'est-à-dire les jurèmes produits dans la figure du cercle d'exigence mutuelle.

${ }^{85}$ C'est-à-dire les jurèmes produits dans la figure du cercle d'exigence mutuelle.

${ }^{86}$ C'est-à-dire les jurèmes émanant unilatéralement d'un (ou plusieurs) émetteur(s) à l'égard d'un (ou plusieurs) destinataire(s).
} 
L'inscription de la loi de la tribu, le processus d'initiation, est d'une violence inouï ${ }^{87}$. C'est par la violence que la société dicte et impose sa loi à ses membres. Comment, dès lors, parler de liberté à leur sujet? Une égalité forcée est-elle à même de garantir la liberté ?

On ne saurait nier l'aspect violent de l'initiation. La violence est voulue, la souffrance remplit une véritable fonction : "l'intensité de la souffrance, les initiateurs veillent à ce qu'elle parvienne à son comble. Un couteau de bambou suffirait amplement, chez les Guayaki, à trancher la peau des initiés. Mais ce ne serait pas suffisamment douloureux. II faut donc utiliser une pierre, un peu coupante, mais pas trop, une pierre qui, au lieu de trancher, déchire. Aussi, un homme à l'œil expert s'en va explorer le lit de certaines rivières, où l'on trouve ces pierres à torturer. (...) L'initiation est, indéniablement, mise à l'épreuve du courage personnel, celui-ci s'exprime, si l'on peut dire, dans le silence opposé à la souffrance $»^{88}$.

Cette violence ne procède-t-elle pas d'un véritable formatage des individus, aliénant ainsi leur liberté ? II est certain que les initiés n'ont pas le choix. II n'est pas question pour eux de refuser l'initiation, de refuser de se plier à l'impératif d'égalité. Le sort réservé à Chachugi l'atteste. Le refus de l'État et l'indépendance de la " tribu » sont-ils au prix de la perte de la liberté individuelle ? Faut-il voir dans ce mécanisme la mise en avant d'une liberté collective (celle de la tribu) au détriment d'une liberté individuelle (celle de ses membres) ?

La question de la liberté est, à l'exception de quelques réflexions laconiques, peu abordée dans l'œuvre de Clastres. Lorsqu'il traite du problème contemporain de la croissance démographique, Clastres semble établir un lien de causalité entre absence d'aliénation et liberté : « la condition pour qu'une société soit primitive, c'est-à-dire sans État, finalement une société où il y ait le minimum d'aliénation et par conséquent le maximum de liberté ${ }^{89}$. Les sociétés primitives mettent en effet en place plusieurs mécanismes visant à empêcher l'aliénation de certains hommes à d'autres, la prise de pouvoir individuelle. Le cercle d'exigence mutuelle implique cependant une aliénation de tous à tous: si les sociétés "primitives" parviennent à prévenir l'émergence d'un pouvoir séparé, c'est parce que chacun exige de tous qu'ils respectent certains principes et sait que les autres en attendent autant de lui. Les sociétés à État favoriseraient l'aliénation volontaire des individus et donc l'aliénation d'une part de liberté : « la machine

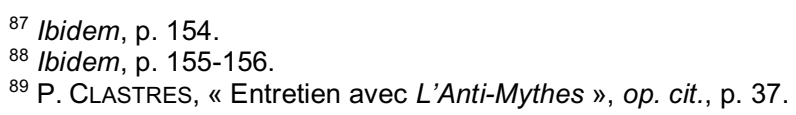


d'État, dans toutes les sociétés occidentales, devient de plus en plus étatique, c'est-à-dire qu'elle va devenir de plus en plus autoritaire ; et de plus en plus autoritaire, pendant un bon moment au moins, avec l'accord profond de la majorité, qu'on appelle le plus souvent la majorité silencieuse ; la majorité silencieuse étant certainement très également répartie à gauche et à droite ${ }^{90}$. Le cercle d'exigence mutuelle qui structure les sociétés sans État n'est-il pas néanmoins la figure la plus emblématique de la servitude volontaire ? Si l'homodoxie s'amenuisait, le cercle ne tarderait pas à se dissoudre ; ce n'est que parce que les membres du cercle maintiennent leurs exigences tout en sachant que les autres en font de même que le cercle se maintient. Si, en outre, un membre du cercle d'exigence mutuelle désirait ne plus y être soumis, il lui suffirait de rompre l'exposition en quittant volontairement le groupe. Dans un épisode de la Chronique que nous rapporterons plus en détail infra, Clastres fait d'ailleurs état de la liberté des Indiens de rompre l'exposition, de quitter le groupe: " s'ils ne pressent pas davantage les compagnons de les suivre, c'est pour respecter leur liberté. Ceux-ci semblent avoir décidé de rester là, il ne faut donc pas les importuner $\iota^{91}$.

Cette liberté qui, pour empêcher l'aliénation de certains hommes à un pouvoir séparé, requiert l'aliénation de chacun à tous les autres peut sembler bien étrange. On s'étonne d'ailleurs que la question ne soit traitée qu'incidemment. Néanmoins, en révisant nos catégories, en interrogeant le sens essentiellement individualiste que l'on donne à la liberté, en relisant le traditionnel Nous homogène à la lumière de la notion de nimbe, une piste émerge : la liberté ne serait-elle pas une notion éminemment conjoncturelle, voire culturelle?

\section{A. Le Nous homogène des sociétés dites primitives: liberté collective versus liberté individuelle}

Puisque «la société primitive fonctionne de telle manière que l'inégalité, l'exploitation, la division y sont impossibles (...), la communauté primitive est à la fois totalité et unité. Totalité, en ce qu'elle est ensemble achevé, autonome, complet, attentive à préserver sans cesse son autonomie, société au sens plein du terme. Unité, en ce que son être homogène persévère dans le refus de la division sociale, dans l'exclusion de l'inégalité, dans l'interdit de l'aliénation. La société primitive est totalité une en ce que le principe de son unité ne lui est pas extérieur : elle ne laisse aucune figure de l'Un se détacher du corps social pour la représenter, pour l'incarner comme

90 Ibidem, p. 38-39.

${ }^{91}$ P. ClastRes, Chronique des Indiens Guyaki, Paris, Plon, 1988, p. 158. 
unité. C'est pourquoi le critère de l'indivision est fondamentalement politique : si le chef sauvage est sans pouvoir, c'est parce que la société n'accepte pas que le pouvoir se sépare de son être, que la division s'établisse entre celui qui commande et ceux qui obéissent. Et c'est aussi pourquoi, dans la société primitive, c'est le chef qui est commis à parler au nom de la société : en son discours, le chef n'exprime jamais la fantaisie de son désir individuel ou le dire de sa loi privée, mais seulement le désir sociologique qu'a la société de rester indivisée et le texte d'une Loi que personne n'a fixée, car elle ne relève pas de la décision humaine. Le législateur est aussi le fondateur de la société, ce sont les ancêtres mythiques, les héros culturels, les dieux. C'est de cette Loi que le chef est porte-parole : la substance de son discours, c'est toujours la référence à la Loi ancestrale que nul ne peut transgresser, car elle est l'être même de la société : violer la Loi, ce serait altérer, changer le corps social, introduire en lui l'innovation et le changement qu'il repousse absolument ${ }^{92}$.

La totalité et l'unité, en un mot l'homogénéité, caractériseraient donc les sociétés dites primitives. Elles formeraient un Nous soudé et unique. La parfaite homogénéité du corps social semble faire obstacle à la conception de la liberté individuelle telle qu'on la conçoit dans les sociétés libérales occidentales. II n'est pas question d'exercer une liberté " qui s'arrête où commence celle des autres $»^{93}$, il est plutôt question d'assurer la parfaite harmonie du corps social. Le mode de production des sociétés "primitives " illustre ce principe. Nous l'avons vu, il est faux de parler d'économie de subsistance. Les sociétés sans État sont des sociétés d'abondance et de loisir: elles consacrent le moins de temps possible à la production de nourriture, elles cherchent à rentabiliser l'effort afin de réduire le temps de travail. Si, d'aventure, un « Sauvage » produisait plus que nécessaire, il ne garderait pas ce surplus mais le partagerait avec la communauté : «l'homme devenu "riche" à la force de son seul poignet verra sa richesse disparaître en

92 P. CLASTRES, Archéologie de la violence. La guerre dans les sociétés primitives, op. cit., p. 4142.

${ }^{93}$ Cette conception est, du reste, consacrée par des textes de première importance dans les ordres juridiques occidentaux contemporains. Ainsi, l'article 4 de la Déclaration des Droits de l'Homme et du Citoyen de 1789 dispose : « La liberté consiste à pouvoir faire tout ce qui ne nuit pas à autrui : ainsi, l'exercice des droits naturels de chaque homme n'a de bornes que celles qui assurent aux autres membres de la société la jouissance de ces mêmes droits. Ces bornes ne peuvent être déterminées que par la loi ". De même, la Déclaration universelle des droits de l'homme adoptée le 10 décembre 1948 dispose, en son article 29 (2) : « Dans l'exercice de ses droits et dans la jouissance de ses libertés, chacun n'est soumis qu'aux limitations établies par la loi exclusivement en vue d'assurer la reconnaissance et le respect des droits et libertés d'autrui et afin de satisfaire aux justes exigences de la morale, de l'ordre public et du bien-être général dans une société démocratique ». 
un clin d'œil entre les mains, ou les estomacs, de ses voisins ${ }^{94}$. II n'est pas question de se développer personnellement. L'enrichissement personnel est d'ailleurs, chez les Guyaki par exemple, structurellement impossible. La " marchandise » principale est la viande. Or, un chasseur ne peut consommer son propre gibier sous peine de devenir pane, c'est-à-dire de s'attirer la malchance à la chasse et, par conséquent, de se placer en marge de la société. "Je tue un animal, mon épouse le découpe, car cela m'est interdit. Elle conserve pour elle-même et les enfants quelques morceaux, et le reste est distribué aux compagnons : d'abord à la parenté, frères et beaux-frères, puis aux autres ${ }^{95}$. "Un chasseur, en somme, passe sa vie à flécher pour les autres et à consommer leur propre gibier. Sa dépendance est totale, comme celle des compagnons par rapport à lui. Les choses sont donc égales, nul n'est jamais lésé puisque tous les hommes "produisent" d'équivalentes quantités de viande. C'est ce qu'on appelle pepy, l'échange ${ }^{96}$. L'échange assure la primauté du groupe sur l'individu, rend l'individu dépendant du groupe. Il en va de sa survie.

L'absence d'égalité est inconcevable. Comment dès lors parler de liberté individuelle? L'individualité, la subjectivité ne peuvent être pensées que par rapport au Nous commun. La notion même d'individualité, laquelle suppose l'unité de l'individu ${ }^{97}$, semble ne pas exister.

Paradoxalement, on trouve dans la Chronique quelque relent d'une conception individualiste de la liberté. Clastres décrit une expédition à la recherche d'un groupe d'Indiens dont l'absence prolongée devenait inquiétante. L'auteur et quelques Aché retrouvèrent le groupe disparu gisant sur le sol, fiévreux, sans doute grippés. "À ma grande surprise - écrit Clastres - les hommes que j'ai suivis restent là dix minutes au plus. Ils n'insistent pas du tout pour que les autres se lèvent ; ramassant leurs armes, ils reprennent le chemin du retour : la mission est remplie, ils les ont trouvés, leur ont parlé ; les compagnons ne veulent pas venir, on s'en retourne donc. On pourrait penser à une indifférence profonde de leur part, à une totale insensibilité quant au sort des malades, voire à de la cruauté. Mais ce n'est pas cela ; en réalité, s'ils ne pressent pas davantage les compagnons de les suivre, c'est pour respecter leur liberté. Ceux-ci semblent avoir décidé de rester là, il ne faut donc pas les importuner. (...) Comme nous commençons

\footnotetext{
${ }^{94}$ P. CLASTRES, Archéologie de la violence. La guerre dans les sociétés primitives, op. cit., p. 40. ${ }^{95}$ P. ClASTRES, Chronique des Indiens Guyaki, op. cit., p. 231.

${ }^{96}$ Ibidem.

${ }^{97}$ On souligne l'incompatibilité de cette unité avec la conception que les Indiens ont d'euxmêmes : à la fois hommes et dieux, cette existence multiple nécessite la médiation du groupe et, à la fois, assure son unité (voy. supra).
} 
à faire marche arrière, le vieux Tokangi se tourne vers le tapy et crie : "Quand vous serez morts, briku u pa modo, les vautours vont vous dévorer !" Perspective répugnante pour les Indiens de ne point quitter le monde des vivants selon les rites : tout, plutôt que d'offrir son cadavre à la nature et à ses animaux, surtout aux vautours. "On vient !" entendons-nous simplement ${ }^{98}$.

Comment expliquer la liberté reconnue aux Indiens quand il s'agit de rester seul pour mourir et l'inévitable contrainte des scarifications lors de l'initiation ? Notre hypothèse tient à l'équilibre du cercle d'exigence mutuelle. En refusant l'initiation, Chachugi perturbait la symétrie des relations entre Indiens, elle se plaçait en dehors du cercle et, dès lors, risquait de n'être ni consciente, ni exposée aux divers agrégats. En restant sur place pour mourir, le groupe de chasseurs ne perturbait en rien l'équilibre social de la tribu. Ils sortaient certes du cercle d'exigence mutuelle mais naturellement, sans persister en dehors. La liberté individuelle n'existerait, dès lors, que dans la mesure où elle ne perturbe pas le fonctionnement du groupe. Cette notion n'est cependant pas suffisamment traitée dans l'œuvre de Pierre Clastres pour sortir de la sphère des hypothèses.

\section{B. La relativité du Nous homogène, le nimbe et le cercle d'exigence mutuelle}

La très forte cohésion du groupe couplée à la liberté laissée aux Indiens quand ils posent des actes qui n'engagent qu'eux-mêmes semble mener à un paradoxe : la liberté individuelle est assurée tant que le groupe y consent. Curieuse conception pour un esprit occidental.

Ce paradoxe se résout peut-être grâce à la notion de nimbe. Le Nous n'est pas inébranlable, il requiert un climat favorable. Si ce climat se dégrade suffisamment, l'égoïsme refait surface et la fiction du groupe comme Nous homogène s'effrite. Le Nous, en ce qu'il contribue à assurer le fonctionnement optimal du cercle d'exigence mutuelle par une fiction, est un nimbe. Une fois ce nimbe dissipé, il importe de ne pas perdre de vue que la figure du cercle d'exigence mutuelle est un tout composé de multiples parties. Si l'homogénéité du groupe s'amenuise, les commandements émis par les membres du groupe, eux, subsistent.

Les sociétés « primitives » sont mues par un idéal autarcique : produire le minimum pour vivre - et non survivre - mais ni plus, ni moins. "L'idéal d'autarcie économique est en fait un idéal d'indépendance politique, laquelle

${ }^{98}$ Ibidem, p. 158. 
est assurée tant que l'on n'a pas besoin des autres ${ }^{99}$. "Cette volonté est aussi à l'œuvre en un sens à l'intérieur de la communauté, où les tendances centrifuges poussent chaque unité de production, chaque "maisonnée", à proclamer : chacun pour soi ! Naturellement, un tel principe, féroce en son égoïsme, ne trouve que rarement à s'exercer : il y faut des circonstances exceptionnelles, comme cette famine dont Firth observa les effets dans la société tikopia, victime en 1953-54 d'ouragans dévastateurs ${ }^{100}$. $\mathrm{Si}$, dans un premier temps, les Tikopia semblaient "s'unir dans l'adversité », Firth observa " un second mouvement de rétractation, un retour à l'isolement domestique, au fur et à mesure que l'adversité devenait désastre ${ }^{101}$. Le « Nous, les Tikopia » se trouva affaibli par la crise, par la fin des conditions optimales au maintien du nimbe. "La maisonnée apparut comme la forteresse de l'intérêt privé, celui du groupe domestique, une forteresse qui en cas de crise s'isole du monde extérieur, relève les ponts-levis sociaux lorsqu'elle ne s'emploie pas à piller les jardins de ses parents et voisins ${ }^{102}$.

La relativité de l'homogénéité du Nous des sociétés dites primitives trouve encore une illustration particulièrement éloquente dans la normativité proverbiale des Maori de Nouvelle-Zélande. L'usage des proverbes y était plus répandu que dans nos sociétés policées. Au lieu d'exprimer un avis personnel, les Maori utilisaient souvent une formule préétablie, un proverbe. La référence à des formules issues de la tradition, attribuées à de glorieux ancêtres (quitte à y faire référence dans le seul but d'en renforcer l'autorité ${ }^{103}$ ), concises et standardisées, permettait à l'opinion publique de s'exprimer d'une voix plus ou moins homogène. C'est elle qui assurait la sanction quand la norme formulée dans un proverbe était transgressée : " the power of public opinion was a very real factor in Maori life. No one was strong enough to defy the whole community and go on his own way regardless of what his companions, neighbours, and kinsmen thought and said. His existence would be rendered absolutely intolerable. (...) Any real defiance of the censure of the community would mean an automatic severance of all those reciprocal relations of an economic and social kind which alone rendered life possible ${ }^{104}$. Curieusement, il existait des proverbes antithétiques qui, pour

\footnotetext{
${ }^{99} \mathrm{P}$. CLASTRES, préface à M. SAHLINS, Âge de pierre, âge d'abondance. L'économie des sociétés primitives, op. cit., p. 17.

$100 \mathrm{Ibidem}$.

101 M. SAHLINS, Âge de pierre, âge d'abondance. L'économie des sociétés primitives, op. cit., p. 177.

102 Ibidem, p. 178.

${ }^{103}$ R. FIRTH, " Proverbs in the Native Life, with Special Reference to Those of the Maori, II », Folklore, 1926, n 3, p. 262.

104 Ibidem, p. 258.
} 
une même situation, mettaient en avant un comportement et son contraire. Ainsi, si l'hospitalité et le don désintéressé étaient socialement encouragés ("Though the present is small it is given through love "; "Those descend from Taorangatira and Turangapeke [figurative names] make presents without looking for any return for them ${ }^{105}$ ), il existait pourtant des proverbes faisant l'apologie de l'égoïsme ("Raw food is still possessed; cooked it goes to another "106 ; "Our food has all vanished from the fast-coming feet of visitors $\left.\aleph^{107}\right)$. Comment expliquer la coexistence de ces directives antagonistes ? II convient de distinguer le comportement idéal du comportement réel. Le comportement idéal est le comportement généreux, travailleur, promu par le groupe et encouragé par des proverbes gratifiants ("Tis the numbers of lightness and ease " ${ }^{108}$; "Well done, O arm so powerful to haul up the large stone which anchors the canoe ${ }^{109}$ ). Le comportement réel est contraire à la moralité exprimée du groupe, il pousse les individus à poursuivre leurs propres intérêts et fait l'objet de commentaires proverbiaux réprobateurs ("Will squatting at home on your haunches bring you food? " ${ }^{110}$; "A dog with a burnt tail ; the man like him » ${ }^{111}$ ). Ces comportements égoïstes sont courants et ont toujours besoin de l'opinion publique pour se réaligner sur l'intérêt du groupe. L'existence de proverbes aux sens opposés dont l'opinion publique ne cautionne que la face collective révèle la fragilité du Nous primitif. Son homogénéité ne dépend que de la capacité du groupe à exercer une pression suffisante sur les individus. Nul penchant naturel à la générosité, nul sentiment exacerbé du collectif, seulement un cercle d'exigence mutuelle dont l'efficacité est renforcée par des proverbes, des formules censées exprimer une loi ancestrale ; le caractère ancestral étant pourvoyeur d'une légitimité particulière. C'est un nimbe. On est loin - et c'est sans doute heureux - du mythe du bon sauvage.

Chez les Guyaki, les hommes se rassemblent la nuit dans la forêt pour chanter. Se saisissant du langage, ils inventent leur propre mythe; ils accèdent, pour un moment, à la réalisation d'eux-mêmes que la société rend structuralement impossible. Seul le langage « peut remplir la double mission

\footnotetext{
105 Ibidem, p. 249.

106 " Meaning, "Eat your food while it is still underdone; when it is cooked visitors may eat it for you" » (ibidem, p. 249).

107 Ibidem.

108 R. FIRTH, "Proverbs in the Native Life, with Special Reference to Those of the Maori, I ", Folklore, 1926, $\mathrm{n}^{\circ}$ 2, p. 138.

109 Ibidem, p. 140.

110 Ibidem, p. 141.

111 "Meaning "a lazy dog sticks so close to the fire that his tail gets singed; this fellow is about as lazy" » (ibidem).
} 
de rassembler les hommes et de briser les liens qui les unissent ${ }^{112}$. Si l'Occident a rendu le langage complétement extérieur à l'homme en ce qu'il est un moyen de communication, "les cultures primitives au contraire, plus soucieuses de célébrer le langage que de s'en servir, ont su maintenir avec lui cette relation intérieure qui est déjà en elle-même alliance avec le sacré ${ }^{113}$. Et comme inconsciemment, ces voix, seule possibilité d'exprimer une réalisation individuelle, " se croisent, à leur insu, en un dialogue qu'elles voulaient oublier ${ }^{114}$. Cette dialectique du rassemblement et du bris rend peut-être compte de la manière dont est vécue la liberté dans ces sociétés : dans la relation singulière qu'ils entretiennent avec leur part divine, les Indiens sont libres et cette liberté les reconduit nécessairement au groupe et à ses traditions, lesquels permettent à la part divine de chacun d'exister.

Du point de vue de la théorie microscopique du droit, si le nimbe peut certes expliquer certaines choses, il convient de ne pas perdre de vue la tension qui caractérise tout cercle d'exigence mutuelle. Celui-ci met nécessairement en jeu une articulation délicate entre le tout et ses parties. Si la pression par menace de sanction qui émane des individus qui composent le groupe est efficace, c'est grâce à l'homogénéité du commandement, laquelle s'explique par une homodoxie donnée. Symétriquement, si chaque individu a les mêmes attentes normatives, c'est grâce à la pression exercée par le groupe. La liberté de l'individu est, dès lors, dans cette configuration, nécessairement aliénée à celle du groupe. Le point de vue positiviste que nous adoptons nous oblige toutefois à rejeter tout réalisme ${ }^{115}$ : les normes, en effet, ne le sont pas par essence mais trouvent leur fondement dans une action humaine (l'humain dût-il justifier sa conduite et ses attentes par des références surnaturelles). Dès lors, si la force de l'homodoxie faiblit et que, par conséquent, les membres du cercle d'exigence mutuelle cessent d'émettre les mêmes jurèmes, l'agglutination de ces jurèmes au niveau du groupe ne sera plus possible. À une structure qui rendait impossible la prise de pouvoir d'un individu isolé, succédera une multitude d'archèmes, c'est-àdire, une multitude de lieux du pouvoir. Si le pouvoir vient du groupe, c'est, en dernière instance, uniquement grâce aux individus qui le composent.

Clastres rend compte d'un entretien qu'il a mené avec un Guarani en 1965. « Cet Indien - écrit Clastres - était un sage, dirigeant spirituel reconnu

\footnotetext{
112 P. ClAStRES, La Société contre l'État, op. cit., p. 109.

113 Ibidem.

114 Ibidem, p. 88.

${ }^{115}$ Au sens qu'a ce concept dans le cadre de la querelle des universaux, c'est-à-dire en tant qu'il s'oppose au nominalisme.
} 
des siens ${ }^{116}$. Alors que les membres du groupe sont désormais contraints de travailler ponctuellement et d'entrer en relation avec le monde blanc, ce sage a bien senti le péril que constituait ce contact pour la sociabilité de la " tribu ». II appelle les siens à respecter leurs rites, lesquels assurent le contact avec les dieux et, nécessairement, avec la part divine des Indiens qui assure la cohésion du groupe. En d'autres termes, il appelle le groupe à rester centré sur lui-même. L'égalité des composantes du cercle d'exigence mutuelle (leur commune divinité) et l'indépendance de celui-ci par rapport à l'extérieur est la condition nécessaire de sa subsistance, de sa liberté d'exister selon sa propre culture :

Beaucoup de nations jouent sur la terre. Ne vous impatientez pas avec elles! Vous autres, continuez à danser! Agitez avec force votre hochet de danse. Que vos sœurs vous accompagnent avec le bâton de danse. Qu'elles sachent le manier ! vos sœurs ! Entonnez bien, sans vous tromper, les chants que vous a inspirés Tupan. Recueillez-les pour vos sœurs : c'est ainsi seulement qu'elles le sauront. Si vous ne recueillez pas ces chants, si vous vous impatientez, si la persévérance vous fait défaut, si vous vous impatientez contre votre propre corps, alors vous n'acquerrez pas la force.

Qu'il continue à se dresser, l'urucu imparfait! Que de cet urucu s'adornent les femmes : et non pas avec les ornements des hommes blancs! Car nous autres devons rester à part.

Les hommes blancs nous ne les supportons pas sur cette terre laide !117

\section{Conclusion : la société ou l'État comme aliénation suprême ?}

«Le monde blanc est tel que l'égalité y est impossible ${ }^{118}$. Le monde blanc, c'est le monde étatique. Sans être des sociétés sans droit, les sociétés étudiées par Clastres se structurent contre le droit étatique. L'émergence du juridique hors du cercle d'exigence mutuelle fondé sur la loi des Ancêtres supposerait nécessairement une relation de domination. Jouant sur plusieurs facteurs, les Indiens empêchent cette émergence : la chefferie permet de canaliser le pouvoir, l'échange du gibier assure la dépendance de l'individu par rapport au groupe et, surtout, la loi du groupe interdit l'inégalité.

Cette égalité imposée est perçue comme une liberté : la société acéphale présente une forte homodoxie, elle se présente comme un Nous

\footnotetext{
${ }^{116}$ P. ClAstres, Le Grand parler..., op. cit., p. 123.

117 Ibidem, p. 135.

${ }^{118}$ P. ClASTRES, Chronique des Indiens Guyaki, op. cit., p. 83.
} 
homogène. Cette liberté est néanmoins fort relative quand son exercice perturbe le fonctionnement du groupe, quand le nimbe de l'homogénéité faiblit et, plus largement, quand les composantes du cercle d'exigence mutuelle du groupe entrent en contact avec d'autres formes de pouvoir. Société fondée sur une loi ancestrale, société contre l'État et, nécessairement, société contre le changement et l'hétérodoxie.

L'État est, quant à lui, caractérisé par le jurème de suprématie, qui ne peut se limiter à un cercle d'exigence mutuelle. Le champ d'application de ce jurème est en effet d'ordre territorial et non personnel. Tant qu'ils maintiennent le champ du juridique dans un cercle d'exigence mutuelle, les Indiens sont donc prémunis contre l'État. Le sacrifice de la liberté individuelle au profit de la liberté du groupe permet à cette dernière de subsister et aux Indiens de maintenir leur mode de vie : ce sacrifice de ce que nous percevons comme leur liberté individuelle leur permet d'assurer la subsistance du cercle et donc, la liberté collective.

Les deux modèles - étatique et non étatique - impliquent donc toujours une certaine aliénation de la liberté individuelle. Quelle part sacrifier? En quelle proportion? Le choix des Indiens semblait clair. Clair du moins tant qu'ils n'avaient pas conscience de la possibilité d'un autre modèle, car c'est au contact de l'extérieur que les Guyaki ont disparu. Mais ceci dépasse notre horizon. 